\title{
Amount, Type, and Timing of Domain-Specific Moderate to Vigorous Physical Activity Among US Adults
}

\author{
Pedro F. Saint-Maurice, David Berrigan, Geoffrey P. Whitfield, Kathleen B. Watson, Shreya Patel, \\ Erikka Loftfield, Joshua N. Sampson, Janet E. Fulton, and Charles E. Matthews
}

\begin{abstract}
Background: Surveillance of domain-specific physical activity in the United States is lacking. Thus, the authors describe domain-specific moderate to vigorous physical activity (MVPA) in a nationwide sample of US adults. Methods: Participants from the AmeriSpeak panel $(\mathrm{n}=2649 ; 20-75$ y; 50\% female) completed the Activities Completed Over Time in 24-Hours previous-day recall. The authors estimated average MVPA duration (in hours per day) overall and in major life domains by sex, age, race/ethnicity, and education. They also described the most commonly reported MVPAs and timing of MVPA during the day. Results: Across all life domains, participants reported an average of 2.5 hours per day in MVPA. Most MVPA was accumulated during work ( $50 \%$ of total, $1.2 \mathrm{~h} / \mathrm{d})$ and household activities $(28 \%, 0.7 \mathrm{~h} / \mathrm{d})$ with less MVPA reported in leisure time $(15 \%, 0.4 \mathrm{~h} / \mathrm{d})$. Time reported in MVPA varied by sex, and race/ethnicity $(P<.05)$. Walking at work and for exercise, childcare, and walking for transportation were the most commonly reported domain-specific MVPAs. A greater proportion of MVPA took place in the morning $(\sim 06: 00 \mathrm{~h})$ and evening $(\sim 18: 00 \mathrm{~h})$. Conclusions: Work and household activities accounted for $78 \%$ of overall MVPA reported, while leisure-time MVPA accounted for only $15 \%$ of the total. Encouraging MVPA during leisure time and transportation remain important targets for promoting MVPA in US adults.
\end{abstract}

Keywords: work, household, leisure, ACT24

Physical activity guidelines recommend adults accumulate at least 150 to 300 minutes per week of moderate-intensity aerobic activity, 75 to 150 minutes per week of vigorous-intensity activity, or an equivalent combination of both. ${ }^{1}$ Adults can achieve these recommendations by engaging in moderate to vigorous physical activity (MVPA) during leisure time, at work, doing household chores, or for transportation to/from places. Participation in leisuretime physical activity has been well characterized in many surveillance studies ${ }^{2-8}$; however, it is less clear how much MVPA US adults accumulate in other domains of living, and what type of activities are most common.

Physical activity surveillance in the United States has focused on the amount and type of leisure-time activities. ${ }^{9}$ These efforts suggest adults spend an average 19 to 25 minutes per day in leisuretime physical activity ${ }^{4,10}$ and typically engage in walking-, sports-, or dance-related activities. ${ }^{2,6}$ National estimates of physical activity in other domains are also available for transportation ${ }^{11}$ but still relatively lacking for work and household activities. National estimates of physical activity in these domains are limited to the National Health and Nutrition Examination Survey that relies on a combined estimate of occupational/household activity ${ }^{12}$ and the American Time Use Survey (ATUS), which does not capture detailed occupational information and may be limited in capturing activity intensity. ${ }^{13}$ Expanding surveillance tools to capture domain-specific activity to include occupational and household

Saint-Maurice, Patel, Loftfield, Sampson, and Matthews are with the Division of Cancer Epidemiology and Genetics, National Cancer Institute, Rockville, MD, USA. Berrigan is with the Division of Cancer Control and Population Sciences, National Cancer Institute, Bethesda, MD, USA. Whitfield, Watson, and Fulton are with the Division of Nutrition, Physical Activity, and Obesity, National Center for Chronic Disease Prevention and Health Promotion, Centers for Disease Control and Prevention, Atlanta, GA, USA. Saint-Maurice (pedro.saintmaurice@nih.gov) is corresponding author.
MVPA is important since activity in these domains can contribute to the health benefits of physical activity. ${ }^{1}$ One key limiting factor for generating US-based domain-specific MVPA estimates has been the reliance on standardized frequency and duration questions in national surveys to assess moderate-longer time periods (eg, typical week or past month). Such standardized survey questions can be less effective for capturing activities scattered over the week or in episodes of different duration. These standard questionnaire-based methods present many challenges to participants when they are asked to parse long periods of recall into domains ${ }^{14}$ or in identifying the timing of when these activities take place during the day. These limitations can be overcome by previous-day recalls since these rely on open-ended reports of specific MVPA and take advantage of the recall benefits of constructing a narrative of the previous day; facilitating the assessment of MVPA across multiple domains of daily living, including household chores, work, and transportation activities. ${ }^{15-19}$

Activities Completed Over Time in 24-hours (ACT24) is an automated online previous-day recall and can be used to estimate the amount, intensity, type, and timing of $175+$ individual activities. ${ }^{20}$ ACT24 was designed to provide a comprehensive assessment of physically active and sedentary behaviors and builds on early and continuing developments of physical activity ${ }^{21}$ and time use research, including the ATUS. Individual activities in ACT24 have been linked to the Compendium of Physical Activities ${ }^{22}$ to estimate the energy cost (metabolic equivalents [METs]) of each activity reported. ACT24 was specifically designed to address previously documented limitations of questionnaire-based assessment methods and ATUS, including a more comprehensive assessment of lower intensity daily activities and body posture, to improve differentiation between physically active and sedentary behaviors, and more detailed coverage of work-related activities. Furthermore, the ACT24 has been designed for large-scale population-based applications and to work on mobile devices, which can 
be an important tool for data collection as these devices become ubiquitous.

In this study, we sought to strengthen our understanding of the epidemiology of MVPA by estimating the amount and type of domain-specific MVPA in a population-based sample of US adults using a novel method for population surveillance, the ACT24 previous-day recall. We specifically describe (1) the amount of physical activity overall and for leisure, work, household chores, transportation, and other purposes; (2) the most common type of activities reported in each domain; and (3) the time of day when domain-specific activities most often occur in the population. Such assessment will advance our understanding of how US adults use their time when being active and inform future physical activity interventions.

\section{Methods}

\section{Study Population and Design}

Participants were from the AmeriSpeak panel, a probabilitybased survey panel that is representative of the US adult population and maintained by the National Opinion Research Center at The University of Chicago. Participants in AmeriSpeak are randomly sampled from the National Opinion Research Center's National Frame an address-based sample that has a combined coverage of $\sim 97 \%$ of US households. AmeriSpeak participants were recruited from this sample frame by US postal mail and upon enrollment were invited over time to take part in a variety of online and telephone surveys. ${ }^{23,24}$ All participant contact and data collection were carried out by research staff at National Opinion Research Center. Data collection took place between October and November of 2019 and informed consent from all study participants was obtained. Participants were asked to complete a short unannounced online survey and an ACT24 recall on a randomly selected day of the week. Those completing this task were then asked to complete a second unannounced ACT24 on a randomly selected day of the week 1 to 2 weeks later.

In this study, we used appropriately weighted data from all available recalls to maximize the range of activities captured on a given day in the population. ACT24 was administered online using a link that was sent via e-mail or text message. Each participant completed the survey and ACT24 on the day that they were assigned. The online survey queried self-rated health, usual physical activity using an adapted online version of the Global Physical Activity Questionnaire, height, and body weight. Data from the survey were merged with ACT24 data and additional demographic information obtained from the AmeriSpeak panel database. Participants received $\$ 30$ for completing the survey and both recalls or $\$ 15$ for completing the survey and only one recall.

\section{Activities Completed Over Time in 24 Hours}

To complete an ACT24 recall (via smartphone, tablet, or computer), participants reported how they spent their time sleeping/ in-bed, being physically active, and in sedentary behaviors on the previous day (midnight-midnight) by selecting from $175+$ individual activities organized into 14 major categories. ${ }^{25}$ After selecting an activity, follow-up questions assessed the duration of the activity, body position, and other details. Addition of activities outside of the predefined list was also possible. Activities reported are linked to the Compendium of Physical Activities ${ }^{22}$ and each activity was scored to estimate energy expenditure using METs. Activities with MET values ranging from 3.0 to 5.9 were classified as moderate intensity while activities with $6.0+$ METs were classified as vigorous intensity. Individual activities were classified according to 5 major domains: Leisure, Work, Household, Transportation, and Other (Supplemental Table S1 [available online]). Data from ACT24 were initially examined for quality and overlaps. When there was more than one activity reported for the same time (ie, overlaps), we prioritized the activity with the highest intensity or MET value, and the activity durations were recalculated. Recalls with $<1$ hour of missing or unknown activity time (ie, gap time, private, or prefer not to say time), and at least 22 hours of total time reported were considered valid. Participants who completed the survey and at least one valid ACT24 recall were included in our analytic sample.

\section{Development of Survey Sample Weights}

The AmeriSpeak panel is primarily based on a stratified 2-stage sampling design. The primary sampling units in the first stage are National Frame Areas, each of which is a combined statistical area, a core-based statistical area that does not belong to any CSA, or a county or county-equivalent geographic unit that does not belong to any core-based statistical area. The secondary sampling units are segments defined from census tracts or block groups. For purposes of variance estimation, the original sampling strata are combined into 47 virtual strata and each stratum is divided into 2 to 122 virtual primary sampling units. To develop the final weights for this study, we started with the final panel weight (eg, a previously calculated weight from the AmeriSpeak panel) and then, for each recall, we calculated study-specific weights that further adjust for selection probabilities from the panel, survey nonresponse in our study, and population coverage. The final weights are ranked to external population totals associated with age, sex, education, race/ethnicity, housing tenure, telephone status, and Census Division derived from the current population survey. ${ }^{23}$ Importantly, we calculated study-specific sampling weights for each day of the week separately (eg, the participants who completed the recall on a Monday are weighted to represent the US population) and then further normalized the weights so each day contributed equally to the analyses (ie, after weighting, each day of the week has an equal number of recalls).

\section{Statistical Analysis}

We calculated descriptive statistics, that is, weighted mean and standard error (SE) for continuous variables and frequency, and weighted percentage for categorical variables. Descriptive statistics were calculated for the sample overall and by sex for demographic factors, including age, race/ethnicity, education, income, and occupational status, as well as body mass index and overall activity level/compliance with aerobic physical activity guidelines (ie, $\geq 150 \mathrm{~min} / \mathrm{wk}$ of moderate-intensity aerobic activity; $\geq 75 \mathrm{~min} /$ wk of vigorous-intensity activity; or a combination of both) determined using information from the Global Physical Activity Questionnaire.

Next, we characterized the distribution of the amount (in hours per day), type (ie, individual activities), and timing (ie, hour of day) for both total and domain-specific MVPA (3.0+ METs). Results for moderate versus vigorous intensity are also provided. However, 
vigorous activity contributed little to total MVPA; hence, our results focus on MVPA. The amount of total MVPA reported was first estimated for the total sample and then only among those who reported any MVPA on the given day. We provided both means and quantiles of MVPA duration (ie, 25th, 50th, and 75th percentiles); we used the Mann-Whitney $U$ and Kruskal-Wallis nonparametric tests to examine associations between the total and domain-specific MVPA and sex (men, women), age (20-34, 35-54, 55-64, or $65+y$ ), race/ethnicity (white non-Hispanic, black non-Hispanic, Hispanic, Asian, or other), and educational attainment (high school or less, some college/associate degree, bachelor's degree, or graduate degree). The amount of domain-specific MVPA was computed to illustrate how each domain contributed to the total MVPA reported. We also calculated Pearson correlations between MVPA overall and specific domains to explore whether respondents more active in one domain were also active overall and in other domains. Physical activity levels can vary between workdays and nonworkdays. Therefore, we also used nonparametric tests to examine differences in the amount of MVPA between those who were employed (separately for work and nonwork days) and those who were unemployed or retired. We identified the most commonly reported types of MVPAs within each of the domains (Supplemental Table S2 [available online]) by estimating the prevalence of the individual MVPA types reported in each domain. Finally, we described the timing of activities over the previous day by plotting the proportion of activities by minute of the day from 00:00 to 23:59 for each domain using a kernel smoother.

All descriptions and analyses were done in SAS (version 9.4; SAS Institute, Cary, NC) using survey analysis procedures (eg, proc surveymeans, proc surveyfreq, etc) accounting for both the sample weights and study design. Associations between MVPA and demographic factors were considered statistically significant if $P$ values were $<.05$.

\section{Results}

A total of 2877 participants, out of 15,153 invited AmeriSpeak panelists, completed the online survey and at least one valid ACT24 recall. Overall, the completion rate was $19 \%$ (2838 participants had first recalls and 1737 had second recalls). Overall, 6.4\% $(n=293)$ and $2.0 \%(n=91)$ of recalls were excluded due to more than 1 hour per day of unknown time and $<22$ hours of total reported activities, respectively. The final analytical sample included 4191 recalls (2499 first recalls and 1692 second recalls) from 2649 participants. Approximately $58 \%$ of participants completed 2 ACT24 recalls. For all demographic characteristics except income, differences between our weighted sample and the US adult population in 2019 were small ( $<5 \%$; Supplemental Table S3 [available online]). For income, our analytical sample included more adults with a lower income $(<\$ 75,000$ per year; $62 \%$ vs $46 \%)$ and fewer with a higher income ( $\geq \$ 125,000$ per year; $14 \%$ vs $28 \%)$.

Participants in the weighted sample averaged 45.2 years old and about $50 \%$ were women (Table 1). About $60 \%$ to $70 \%$ of the participants were non-Hispanic white, completed high school or some college, reported income of $<\$ 99,000$, and were employed at the time of the study. Approximately $35 \%$ of participants reported a body mass index $\geq 30.0 \mathrm{~kg} / \mathrm{m}^{2}$ and $58 \%$ reported meeting aerobic physical activity guidelines.

About $70 \%$ of participants reported participating in some MVPA on a given day, with an average total duration of 2.5 hours per day and median duration of 1.0 hour per day across all domains. The amount of MVPA reported across all domains varied by sex and race/ethnicity. Men reported significantly more MVPA than women $(2.9 \mathrm{~h} / \mathrm{d}$ vs $2.1 \mathrm{~h} / \mathrm{d} ; P<.01)$ and the amount of MVPA per day was associated with race/ethnicity $(P<.01)$. There were no significant associations between the total amount of MVPA and age $(P=.62)$ or educational attainment $(P=.35)$. Among participants reporting some MVPA $(\mathrm{n}=2092)$, the average amount of reported MVPA was 3.6 hours per day (Table 2). Approximately $85 \%$ of total MVPA was accumulated in moderate-intensity activities with little time reported in vigorous activities (Supplemental Table S4 [available online]).

Examination of domain-specific MVPA revealed that the majority of daily MVPA was accumulated during work (50\% of total MVPA; $1.2 \mathrm{~h} / \mathrm{d}$ ) and household-related activities (28\% of total MVPA; $0.7 \mathrm{~h} / \mathrm{d})$. Participants reported less MVPA during leisure time $(15 \% ; 0.4 \mathrm{~h} / \mathrm{d})$ and very little MVPA for transportation $(2 \%$; $<0.1 \mathrm{~h} / \mathrm{d}$; Figure 1; Supplemental Table S5 [available online]). The amount of leisure-time and work-related MVPA was higher among men compared with women $(P<.01)$. The amount of leisure-time MVPA was positively associated with age $(P=.03)$ and education $(P<.01)$. Time reported in work-related activities was inversely associated with age $(P<.01)$ and education $(P<.01)$. Amount of MVPA at work was also associated with race/ethnicity $(P=.01)$. Household MVPA was significantly associated with race/ethnicity $(P<.01)$ but did not vary across other demographic characteristics $(P>.05)$. Transportation MVPA varied across all demographic factors except by sex $(P=.54)$ (Figure 1; Supplemental Tables S6-S9 [available online]). The total amount of MVPA per day was positively correlated with leisure $(r=.2)$, work $(r=.8)$, household chores $(r=.3)$, and other activities $(r=.3)$. Correlations between domain-specific MVPA estimates were low (all $r<.1$; Supplemental Table S10 [available online]).

The average amount of MVPA reported was considerably higher on workdays $(n=1469 ; 3.3 \mathrm{~h} / \mathrm{d})$ when compared with nonworkdays ( $\mathrm{n}=471 ; 1.8 \mathrm{~h} / \mathrm{d})$ or when compared with participants who were unemployed/retired $(\mathrm{n}=709 ; 1.9 \mathrm{~h} / \mathrm{d} ; P<.01)$. The majority of MVPA during workdays was accumulated during work (76\%), while MVPA on nonworkdays or among unemployed/retired participants was accumulated primarily in household (47\%-56\%) and leisure-time activities (21\%-28\%) (Supplemental Table S11 [available online]).

Next, we examined the most prevalent types of MVPAs reported in each domain. Among the 5 most reported leisure-time MVPAs, we found that walking for exercise was the most frequently reported activity (39.4\% of total leisure-time activity instances), followed by strength and conditioning (16.7\%), cardio (10.7\%), running $(8.9 \%)$, and cycling $(7.7 \%)$. Childcare $(30.3 \%)$, work in the house $(25.1 \%)$, and gardening $(18.3 \%)$ represented most householdrelated MVPAs while pet care and home maintenance contributed with modest proportions for the total activities done in this domain. Most of the activities done for work or transportation involved standing or walking-related activities $(73 \%-82 \%$ of total activities in these domains) (Figure 2; Supplemental Table S12 [available online]).

Our exploration of the timing of domain-specific activity showed that most MVPA took place between 06:00 and 18:00 hours with no clear differences by domain in the overall population. Workrelated activities tended to peak early in the morning with about $60 \%$ of activities done in this domain taking place before noon. Leisure, household, and transportation activities had similar occurrences throughout the day with peaks either early in the morning or late in the evening, possibly mimicking before/after work hours (Figure 3; Supplemental Table S13 [available online]). 
Table 1 Descriptive Characteristics by Sex, US Adults Aged 20-75 Years

\begin{tabular}{|c|c|c|c|}
\hline & All $(N=2649)$ & Men $(n=1464)$ & Women $(n=1185)$ \\
\hline & Mean (SE) & Mean (SE) & Mean (SE) \\
\hline \multirow[t]{2}{*}{ Age, y } & $45.2(0.5)$ & $46.4(0.7)$ & $44.1(0.7)$ \\
\hline & $\begin{array}{c}\text { Frequency } \\
\text { (weighted \%) }\end{array}$ & $\begin{array}{c}\text { Frequency } \\
\text { (weighted \%) }\end{array}$ & $\begin{array}{c}\text { Frequency } \\
\text { (weighted \%) }\end{array}$ \\
\hline \multicolumn{4}{|l|}{ Age, $y$} \\
\hline $20-34$ & $865(32.4)$ & $400(28.4)$ & $465(36.3)$ \\
\hline $35-54$ & $1027(35.2)$ & $602(36.2)$ & $425(34.1)$ \\
\hline $55-64$ & $473(19.2)$ & $286(20.5)$ & $187(17.8)$ \\
\hline $65+$ & $284(13.3)$ & $176(14.9)$ & $108(11.7)$ \\
\hline \multicolumn{4}{|l|}{ Race/ethnicity } \\
\hline White, non-Hispanic & $1803(63.8)$ & $1052(66.5)$ & $751(61.1)$ \\
\hline Black, non-Hispanic & $283(10.9)$ & $104(8.4)$ & $179(13.4)$ \\
\hline Hispanic & $338(16.9)$ & 149 (14.6) & $189(19.2)$ \\
\hline Asian & $105(3.7)$ & $82(4.8)$ & $23(2.5)$ \\
\hline Other $^{\mathrm{a}}$ & $120(4.8)$ & $77(5.7)$ & $43(3.9)$ \\
\hline \multicolumn{4}{|l|}{ Educational attainment } \\
\hline High school or less & $384(34.4)$ & $210(36.2)$ & $174(32.5)$ \\
\hline Some college/associate degree & $1027(28.8)$ & $527(26.5)$ & $500(31.1)$ \\
\hline Bachelor's degree & $721(21.2)$ & 424 (21.9) & $297(20.5)$ \\
\hline Graduate degree & $517(15.7)$ & $303(15.4)$ & $214(15.9)$ \\
\hline \multicolumn{4}{|l|}{ Household income, \$ } \\
\hline$<50,000$ & $966(41.1)$ & $451(37.2)$ & $515(45.0)$ \\
\hline $50,000-99,000$ & $951(34.5)$ & $556(37.1)$ & $395(31.9)$ \\
\hline $100,000-149,000$ & $449(15.1)$ & $269(15.3)$ & $180(14.9)$ \\
\hline $150,000+$ & $283(9.3)$ & $188(10.3)$ & $95(8.2)$ \\
\hline \multicolumn{4}{|l|}{ Occupational status } \\
\hline Employed & $1940(67.0)$ & $1115(69.9)$ & $825(64.3)$ \\
\hline Unemployed/retired & $709(33.0)$ & $349(30.1)$ & $360(35.7)$ \\
\hline \multicolumn{4}{|l|}{ Body mass index, $\mathrm{kg} / \mathrm{m}^{2}$} \\
\hline$<25.0$ & $798(30.0)$ & $416(29.0)$ & $382(31.0)$ \\
\hline $25.0-29.9$ & 845 (32.6) & $509(34.5)$ & $336(30.7)$ \\
\hline $30.0+$ & $932(35.1)$ & $494(34.2)$ & $438(36.0)$ \\
\hline Missing & $74(2.3)$ & $45(2.3)$ & $29(2.3)$ \\
\hline \multicolumn{4}{|l|}{ Aerobic physical activity ${ }^{\mathrm{b}}$} \\
\hline Inactive & $327(14.0)$ & $171(13.0)$ & $156(15.0)$ \\
\hline Insufficiently active & $756(27.6)$ & $373(24.1)$ & $383(31.1)$ \\
\hline Sufficiently active & 567 (21.6) & $302(19.7)$ & $265(23.6)$ \\
\hline Highly active & $989(36.5)$ & $614(43.2)$ & $375(30.0)$ \\
\hline Missing & $10(0.2)$ & $4(0.0)$ & $6(0.3)$ \\
\hline
\end{tabular}

${ }^{\mathrm{a}}$ Other race/ethnicity includes non-Hispanics reporting Other or 2 or more race/ethnicities. ${ }^{\mathrm{b}}$ Aerobic physical activity levels were based on current aerobic physical activity guidelines. Highly active was defined as $>300$ minutes of moderate-intensity aerobic activity, $>150$ minutes of vigorous-intensity aerobic activity, or an equivalent combination of moderate- and vigorous-intensity physical activity per week. Sufficiently active was defined as 150 to 300 minutes of moderate-intensity aerobic activity, 75 to 150 minutes of vigorous-intensity aerobic activity, or an equivalent combination of moderate- and vigorous-intensity aerobic activity per week. Insufficiently active was defined as some aerobic activity but not enough to meet the highly or sufficiently active definition.

\section{Discussion}

Adults in this study reported accumulating $\sim 80 \%$ of their overall MVPA at work and in household-related activities, with leisuretime MVPA comprising the bulk of the remaining contribution. We found that leisure-time activity contributions to the total amount of MVPA were modest ( $15 \%$ of total). Walking was the most common leisure-time activity reported and MVPA usually took place between 06:00 and 18:00 hours. Using a novel assessment tool for population-based surveillance (ACT24), we showed that activities done during work and while at home are the major contributors to daily MVPA in US adults and described the most commonly reported MVPAs in major life domains and the timing of MVPA during the day. 
Table 2 The MVPA on Randomly Selected Days in All Participants and Those Who Reported Some MVPA (Percentage Reporting, Mean, and Quantiles)

\begin{tabular}{|c|c|c|c|c|c|}
\hline & \multicolumn{3}{|c|}{ All participants $(\mathrm{N}=\mathbf{2 6 4 9})$} & \multicolumn{2}{|c|}{$\begin{array}{l}\text { Participants reporting some } \\
\text { MVPA }(n=2092)^{b}\end{array}$} \\
\hline & Reporting some MVPA & Hours/day & Quantiles & Hours/day & Quantiles \\
\hline & Percentage (SE) & $\overline{\text { Mean (SE) }}$ & 50th (25th, 75th) & Mean (SE) & $\overline{50 \text { th (25th, 75th) }}$ \\
\hline All & $70.0(1.0)$ & $2.5(0.1)$ & $1.0(0.0,3.5)$ & $3.6(0.1)$ & $2.1(0.9,5.3)$ \\
\hline \multicolumn{6}{|l|}{ Sex $*$} \\
\hline Men & $71.6(1.5)$ & $2.9(0.1)$ & $1.3(0.0,4.5)$ & $4.0(0.2)$ & $2.5(1.0,6.1)$ \\
\hline Women & $68.4(1.5)$ & $2.1(0.1)$ & $0.8(0.0,2.7)$ & $3.1(0.1)$ & $1.7(0.8,4.3)$ \\
\hline \multicolumn{6}{|l|}{ Age, y } \\
\hline 20-34 & $70.6(1.9)$ & $2.7(0.2)$ & $1.1(0.0,3.9)$ & $3.9(0.3)$ & $2.1(0.9,6.2)$ \\
\hline $35-54$ & $70.0(1.7)$ & $2.6(0.1)$ & $1.0(0.0,3.8)$ & $3.7(0.1)$ & $2.1(0.9,6.0)$ \\
\hline $55-64$ & $67.6(2.5)$ & $2.2(0.2)$ & $1.0(0.0,3.2)$ & $3.3(0.2)$ & $2.1(0.9,5.0)$ \\
\hline $65+$ & $71.9(2.7)$ & $2.0(0.2)$ & $1.1(0.0,2.8)$ & $2.8(0.2)$ & $1.9(1.0,3.9)$ \\
\hline \multicolumn{6}{|l|}{ Race/ethnicity* } \\
\hline White, non-Hispanic & $70.9(1.1)$ & $2.5(0.1)$ & $1.1(0.0,3.5)$ & $3.5(0.1)$ & $2.2(1.0,5.0)$ \\
\hline Black, non-Hispanic & $60.1(3.5)$ & $2.4(0.2)$ & $0.7(0.0,3.3)$ & $4.0(0.4)$ & $2.2(1.0,7.0)$ \\
\hline Hispanic & $72.1(2.8)$ & $2.5(0.3)$ & $1.0(0.0,3.6)$ & $3.5(0.3)$ & $1.9(0.8,5.4)$ \\
\hline Asian & $68.2(4.8)$ & $1.6(0.3)$ & $0.7(0.0,1.7)$ & $2.4(0.4)$ & $1.1(0.7,2.1)$ \\
\hline Other $^{\mathrm{a}}$ & $73.9(4.7)$ & $3.3(0.4)$ & $1.6(0.0,6.0)$ & $4.5(0.5)$ & $2.9(1.0,8.0)$ \\
\hline \multicolumn{6}{|l|}{ Educational attainment } \\
\hline High school or less & $69.0(2.3)$ & $2.8(0.2)$ & $1.2(0.0,4.2)$ & $4.1(0.3)$ & $2.4(1.0,6.9)$ \\
\hline Some college/associate degree & $68.8(1.5)$ & $2.6(0.1)$ & $1.0(0.0,3.9)$ & $3.8(0.1)$ & $2.1(1.0,6.0)$ \\
\hline Bachelor's degree & $71.7(1.7)$ & $2.2(0.2)$ & $1.0(0.0,3.0)$ & $3.0(0.1)$ & $1.9(0.8,4.4)$ \\
\hline Graduate degree & $72.0(2.1)$ & $2.0(0.1)$ & $0.9(0.0,2.7)$ & $2.8(0.1)$ & $1.7(0.8,3.5)$ \\
\hline
\end{tabular}

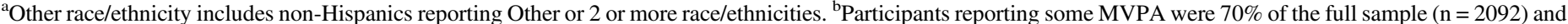

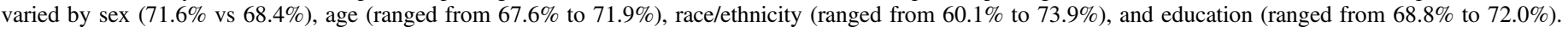

* Statistically significant associations between demographic factor and total MVPA.

Interestingly, we found that leisure-time activities contributed only modestly to total MVPA by an amount $(0.4 \mathrm{~h} / \mathrm{d}$ or $\sim 25 \mathrm{~min} / \mathrm{d})$ that is comparable with results from ATUS and physical activity surveillance reports in the United States. ${ }^{4,10}$ The 2.5 hours per day of total MVPA reported here is higher than many previous estimates based on standardized survey questions. However, these higher estimates are consistent with the ACT24 assessment of activity in all domains, including, work, home, transportation, and leisure-time MVPA. ${ }^{10,14,26-29}$ Studies that include device-based assessments are particularly well suited to clarify the amount of total MVPA in the US population; however, estimates obtained from devices can vary depending on whether the calibration method used only an "ambulatory" protocol or a more comprehensive "ambulatory + lifestyle" calibration protocol. ${ }^{30}$ Device-based estimates of total MVPA in population-based surveys of US adults range from 0.4 to 2.2 hours per day, with larger amounts recorded by devices calibrated in a more comprehensive manner. ${ }^{26,27,30}$ It may be that a more comprehensive assessment of total MVPA, including occupational activity, will yield substantially larger estimates of MVPA duration in the population. Previous-day recalls and devices were designed to measure constructs related to physical activity behaviors; however, the 2 measures use different approaches (eg, recall of individual activities vs recorded movements from devices) and hence comparisons between the 2 ought to be made with caution. ${ }^{31}$ One additional challenge is that MVPA data are positively skewed. ${ }^{32,33}$ In our study, $30 \%$ of respondents reported 0 minutes of activity and the median duration of MVPA was only 1.0 hour per day, substantially lower than our average duration of MVPA $(2.5 \mathrm{~h} / \mathrm{d})$. More research is needed to better understand the distribution of total MVPA in the US population and determine compliance with physical activity guidelines. Information from a variety of measurement methods may be needed to achieve this goal.

Another important feature of our study was the ability to describe the type and timing of activities. Many surveys and surveillance systems collect data only on frequency and duration of activity at a given intensity (eg, National Health and Nutrition Examination Survey, National Health Interview Survey). Some have also collected types of activities performed (eg, 1999-2006 National Health and Nutrition Examination Survey and current Behavioral Risk Factor Surveillance System), but time-use surveys including the ATUS and the ACT24 are distinctive in collecting time, type, and duration of activity across many domains. Results from ACT24 complement past surveillance of time use via the ATUS with improved estimates of activity intensity during work, which was the largest single source of MVPA in the present study. Our study also confirms walking as an important source of MVPA $^{9,34-36}$ reinforcing the importance of understanding how walking or steps per day might be related to health. ${ }^{37}$ Consistent with previous studies, we found that household chores or gardening are common moderate to vigorous activities among US adults. ${ }^{38,39}$ Our timing descriptions showed that a greater proportion of MVPA takes place early in the morning (ie, 06:00-08:00 h) or late in the evening (ie, 18:00 h), which could indicate that MVPA usually 


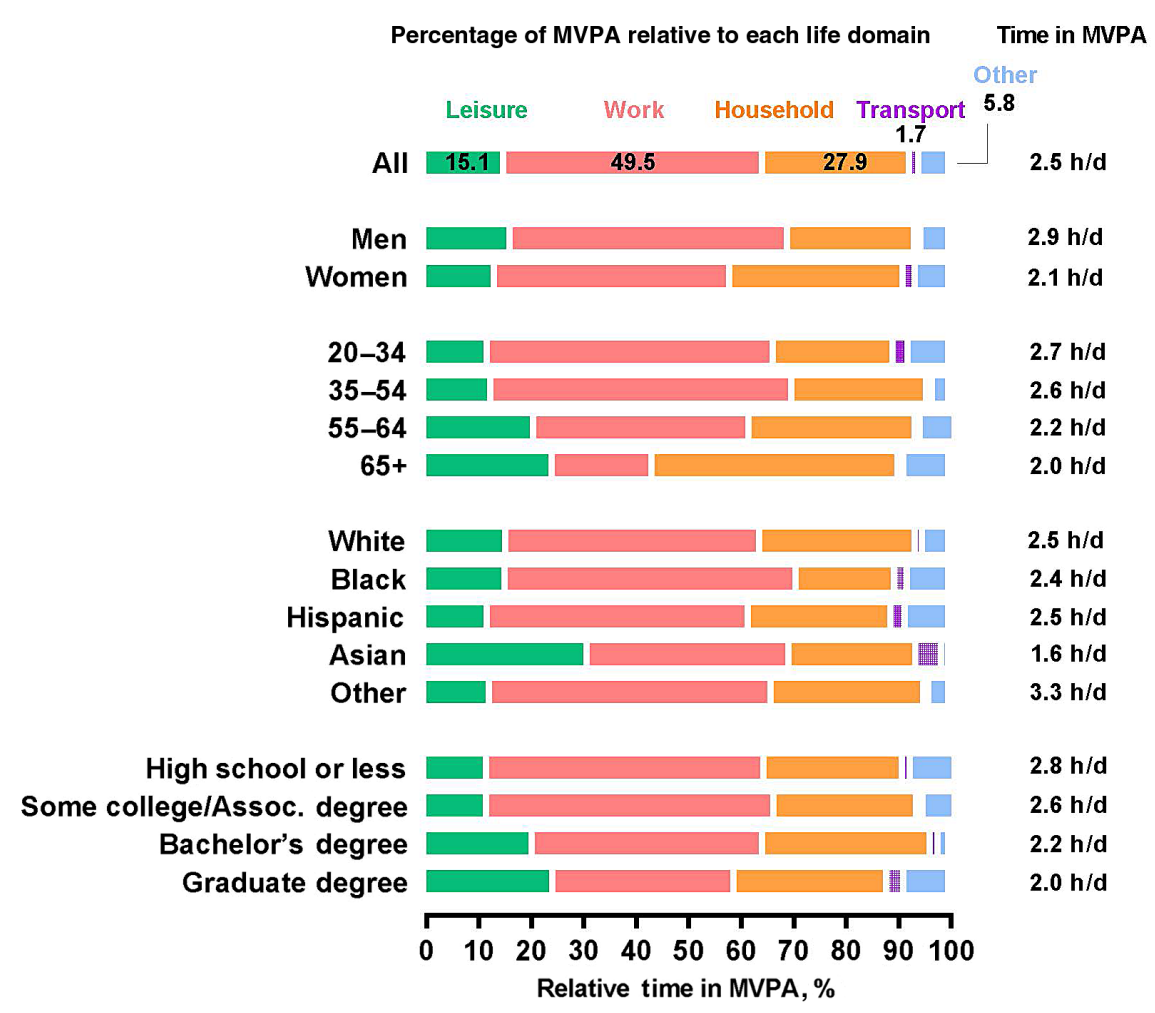

Figure 1 - Relative amount in domain-specific MVPA in all participants by sex, age group, race/ethnicity, and educational attainment. Other race/ ethnicity includes non-Hispanics reporting other or 2 or more race/ethnicities. MVPA indicates moderate to vigorous physical activity.

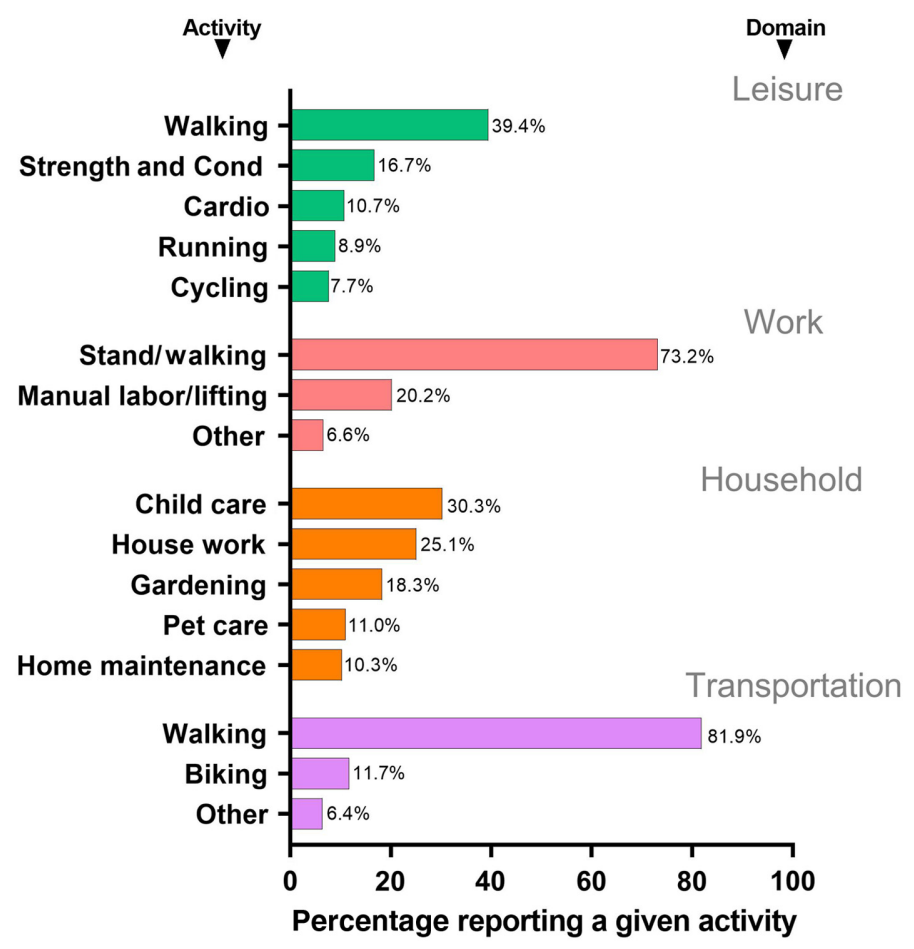

Figure 2 - Top 5 moderate to vigorous physical activity behaviors reported in leisure, work, household chores, and transportation domains. See Supplemental Table S2 (available online) for complete list of activities.

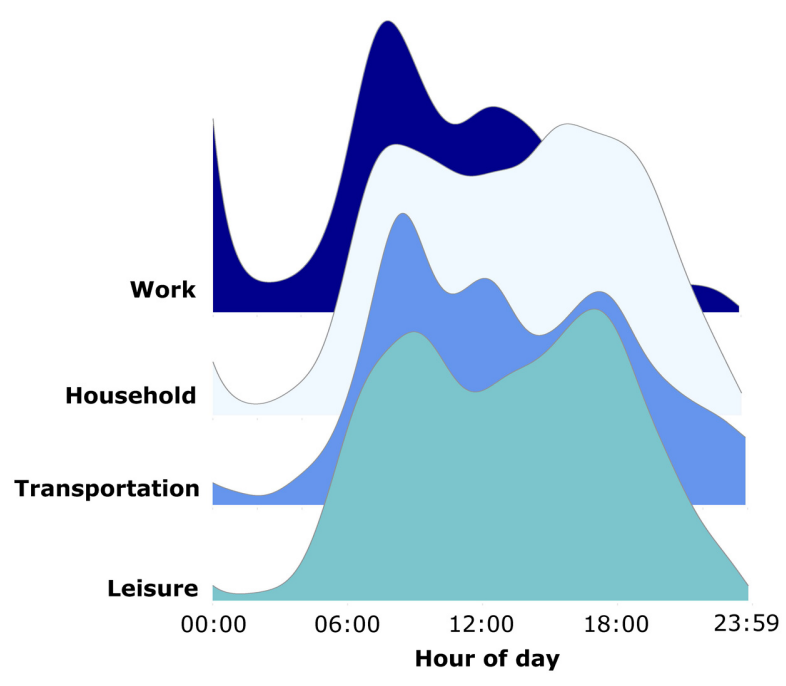

Figure 3 - Timing of moderate to vigorous physical activity by domain. Values are for each domain and represent the percentage of participants among those that reported some MVPA $(n=2092)$ reporting behaviors at a given time of day. 
takes place before or/and after work during workdays. Interestingly, our study shows that there might be important variability in activity levels throughout the day with domain-specific activity peaking at different times in the day.

This study also highlights the potential of web-based previous-day recalls for assessing physical activity in large population studies. The possibility of assigning energy cost to individual activity behaviors has improved the value of previous-day recalls and contributed to the growing interest in these tools. ${ }^{13}$ Previous-day recalls can overcome some of the limitations of questionnaires (eg, Global Physical Activity Questionnaire), including recall bias and incomplete coverage of activities, and may generate more accurate aggregate estimates of activity along with contextual information (eg, purpose of activity). A validation study compared activity estimates obtained from 24-hour recalls to those obtained from wearable cameras clipped to participant's clothes. ${ }^{40}$ The study found that participants accurately reported $\sim 70 \%$ of the activities done in the previous day and that were recorded by the camera. There was a small difference $(4 \mathrm{~min} / \mathrm{d}$ ) between the recall and the camera in the average time spent in leisure-time physical activity $\left(25 \mathrm{~min} / \mathrm{d}\right.$ for the recall vs $21 \mathrm{~min} / \mathrm{d}$ for the camera). ${ }^{40}$ One other study also compared a previous-day recall to directly observe physical activity and found that participants were able to accurately report the location and purpose of active behaviors. ${ }^{41}$ This evidence suggests that previous-day recalls can be used to accurately describe activity in populations. In this study, we demonstrate the potential of ACT24 to describe the various dimensions of physical activity in a large sample of US adults. The domain-specific activity information generated from ACT24 can be used to target domains where there is potential for promoting physical activity. For example, our results show that leisure and transport were the 2 domains with the lowest amounts of MVPA; reinforcing the importance of promoting activity in these settings using established strategies. ${ }^{42}$ Another potential application is to investigate further and document strategies that individuals use to be active at home-a domain where MVPA amounts were among the highest in our study.

There are several limitations to this study. Report-based measures are prone to measurement error and participants in our study could have overreported their activity levels. Overreporting of activity levels has been attributed to social desirability; however, studies that have tested this hypothesis directly found no support for this type of bias in physical activity estimates obtained from previous-day recalls. ${ }^{43,44}$ Participants in our study were asked to report activity levels in the fall of 2019 prior to the COVID-19 pandemic and received an invitation to complete the ACT24 on a random day of the week. This approach allows for generalizations of activity levels during a typical day in the fall of 2019, but this study was not designed to estimate "usual" activity levels over the entire year for individual participants. For example, in the United States, physical activity levels vary throughout the year, with more noticeable changes during the summer. ${ }^{45,46}$ A large proportion of AmeriSpeak panelists opted out of our study (completion rate $=19 \%$ ) and nonparticipation or nonresponse can affect the generalizability of survey results. However, demographics of the sample of respondents who participated were largely comparable with the US population. This study is also limited in that while the scope was to describe activity across the sample, profiles were not characterized in a more detailed manner.
A more in-depth understanding of the nuances of physical activity in each domain is needed to inform professionals and practitioners who implement targeted strategies to increase physical activity.

\section{Conclusions}

We found that US adults report accumulating MVPA across several life domains and that most MVPA was reported during work and household activities. We also found that the most common reported MVPA across the various domains involved walking and that activity was typically done in the morning or evening. ACT24 may be useful for describing the distribution of MVPA in the population and inform future interventions about the domains where activity levels may be improved. Leisure and transportation are 2 potential domains for promoting MVPA in the US adult population.

\section{Acknowledgments}

This research was supported by the Intramural Research Program of the National Institutes of Health, National Cancer Institute. The findings and conclusions in this manuscript are those of the authors and do not necessarily represent the official position of the Centers for Disease Control.

\section{References}

1. United States Department of Health and Human Services. Physical Activity Guidelines for Americans. 2nd ed. Washington, DC: US Department of Health and Human Services; 2018.

2. Woods RA. Sports and Exercise. Washington, DC: US Bureau of Labor Statistics; 2017.

3. Blackwell DL, Clarke TC. State variation in meeting the 2008 federal guidelines for both aerobic and muscle-strengthening activities through leisure-time physical activity among adults aged 18-64: United States, 2010-2015. Natl Health Stat Report. 2018; 112:1-22.

4. Sturm R, Cohen DA. Free time and physical activity among Americans 15 years or older: Cross-sectional analysis of the American time use survey. Prev Chronic Dis. 2019;16:E133. PubMed ID: 31560643 doi:10.5888/pcd16.190017

5. Watson KB, Dai S, Paul P, Carlson SA, Carroll DD, Fulton J. The attributable proportion of specific leisure-time physical activities to total leisure activity volume among US Adults, National Health and Nutrition Examination Survey 1999-2006. J Phys Act Health. 2016; 13(11):1192-1201. doi:10.1123/jpah.2015-0695

6. Watson KB, Frederick GM, Harris CD, Carlson SA, Fulton JE. US adults' participation in specific activities: behavioral risk factor surveillance system-2011. J Phys Act Health. 2015;12:S3-S10. doi:10.1123/jpah.2013-0521

7. Centers for Disease Control and Prevention. Adult participation in aerobic and muscle-strengthening physical activities-United States, 2011. MMWR Morb Mortal Wkly Rep. 2013;62(17):326-330.

8. Ussery EN, Fulton JE, Galuska DA, Katzmarzyk PT, Carlson SA. Joint prevalence of sitting time and leisure-time physical activity among US adults, 2015-2016. JAMA. 2018;320(19):2036-2038. PubMed ID: 30458482 doi:10.1001/jama.2018.17797

9. Piercy KL. Recent trends in adherence of physical activity and sedentary behavior-We need to move more and sit less. JAMA Netw Open. 2019;2(7):e197575. PubMed ID: 31348499 doi:10.1001/ jamanetworkopen.2019.7575 
10. Evenson KR, Wen F, Herring AH. Associations of accelerometryassessed and self-reported physical activity and sedentary behavior with all-cause and cardiovascular mortality among US adults. Am J Epidemiol. 2016;184(9):621-632. PubMed ID: 27760774 doi:10. 1093/aje/kww070

11. Whitfield GP, Paul P, Wendel AM. Active transportation surveillance-United States, 1999-2012. MMWR Surveill Summ. 2015;64(7):1-17. PubMed ID: 26313567 doi:10.15585/mmwr. ss6407a1

12. Whitfield GP, Ussery EN, Carlson SA. Combining data from assessments of leisure, occupational, household, and transportation physical activity among US adults, NHANES 2011-2016. Preventing Chron Diseas. 2020;17:E117. doi:10.5888/pcd17.200137

13. Harms T, Berrigan D, Gershuny J. Daily metabolic expenditures: estimates from US, UK and polish time-use data. BMC public health. 2019;19:453. doi:10.1186/s12889-019-6762-9

14. Whitfield GP, Ussery EN, Saint-Maurice PF, Carlson SA. Trends in aerobic physical activity participation across multiple domains among US Adults, National Health and Nutrition Examination Survey 2007/ 2008 to 2017/2018. J Phys Act Health. 2021;18(suppl):S64-S73. doi:10.1123/jpah.2021-0173

15. Schatzkin A, Subar AF, Moore S, et al. Observational epidemiologic studies of nutrition and cancer: the next generation (with better observation). Cancer Epidemiol Biomark Prev. 2009;18(4):10261032. doi:10.1158/1055-9965.EPI-08-1129

16. Gomersall SR, Norton K, Maher C, English C, Olds TS. In search of lost time: when people undertake a new exercise program, where does the time come from? A randomized controlled trial. J Sci Med Sport. 2015;18(1):43-48. PubMed ID: 24602689 doi:10.1016/j.jsams.2014. 01.004

17. Gomersall S, Maher C, English C, Rowlands A, Olds T. Time regained: when people stop a physical activity program, how does their time use change? A randomised controlled trial. PLoS One. 2015;10(5):e0126665. PubMed ID: 26023914 doi:10.1371/journal. pone. 0126665

18. Dunton GF. Ecological momentary assessment in physical activity research. Exerc Sport Sci Rev. 2017;45(1):48-54. PubMed ID: 27741022 doi:10.1249/JES.0000000000000092

19. Sullivan O, Gershuny J, Sevilla A, Walthery P, Vega-Rapun M. Time use diary design for our times-An overview, presenting a Click-andDrag Diary Instrument (CaDDI) for online application. J Timue Use Res. 2020;15(1):1-17.

20. Division of Cancer Epidemiology \& Genetics NCI. ACT24: Physical activities completed over time in 24 hours. 2021. Accessed December $12,2020$.

21. Matthews CE, Moore SC, George SM, Sampson J, Bowles HR. Improving self-reports of active and sedentary behaviors in large epidemiologic studies. Exerc Sport Sci Rev. 2012;40(3):118-126. PubMed ID: 22653275 doi:10.1097/JES.0b013e31825b34a0

22. Ainsworth BE, Haskell WL, Herrmann SD, et al. Compendium of physical activities: a second update of codes and MET values. Med Sci Sports Exerc. 2011;43:1575. doi:10.1249/MSS.0b013e31821ece12

23. NORC. Technical overview of the AmerSpeak ${ }^{\circledR}$ Panel NORC's probability-based household panel. 2018. http://www.norc.org/ PDFs/AmeriSpeak\%20Technical\%20Overview\%202015\%2011\% 2025.pdf. Accessed Feburary 2, 2019.

24. NORC. AmeriSpeak ${ }^{\circledR}$ Panel Demographics Report. 2018; https:// d3qi0qp55mx5f5.cloudfront.net/amerispeak/i/research/AmeriSpeak_ Panel_Demographic_Report_2018_08_30.pdf?mtime=1535739132. Accessed Feburary 2, 2019.

25. Matthews CE, Kozey Keadle S, Moore SC, et al. Measurement of active and sedentary behavior in context of large epidemiologic studies. Med Sci Sports Exerc. 2018;50(2):266-276. PubMed ID: 28930863 doi:10.1249/MSS.0000000000001428

26. Matthews CE, Keadle SK, Troiano RP, et al. Accelerometermeasured dose-response for physical activity, sedentary time, and mortality in US adults. Am J Clin Nutr. 2016;104(5):1424-1432. PubMed ID: 27707702 doi:10.3945/ajcn.116.135129

27. Welk GJ, Kim Y, Stanfill B, et al. Validity of 24-h physical activity recall: physical activity measurement survey. Med Sci Sports Exerc. 2014;46(10):2014-2024. PubMed ID: 24561818 doi:10.1249/MSS. 0000000000000314

28. Strain T, Wijndaele K, Dempsey PC, et al. Wearable-devicemeasured physical activity and future health risk. Nat Med. 2020;26(9): 1385-1391. PubMed ID: 32807930 doi:10.1038/s41591-020-1012-3

29. Berrigan D, Troiano RP, McNeel T, Disogra C, Ballard-Barbash R. Active transportation increases adherence to activity recommendations. Am J Prev Med. 2006;31(3):210-216. PubMed ID: 16905031 doi:10.1016/j.amepre.2006.04.007

30. Troiano RP, Berrigan D, Dodd KW, Masse LC, Tilert T, McDowell M. Physical activity in the United States measured by accelerometer. Med Sci Sports Exerc. 2008;40(1):181-188. PubMed ID: 18091006 doi:10.1249/mss.0b013e31815a51b3

31. Troiano RP, Stamatakis E, Bull FC. How can global physical activity surveillance adapt to evolving physical activity guidelines? needs, challenges and future directions. Br J Sports Med. 2020; 54(24):1468-1473. PubMed ID: 33239352 doi:10.1136/bjsports2020-102621

32. Welk GJ, Beyler NK, Kim Y, Matthews CE. Calibration of self-report measures of physical activity and sedentary behavior. Med Sci Sports Exerc. 2017;49(7):1473-1481. PubMed ID: 28240704 doi:10.1249/ MSS.0000000000001237

33. Nusser SM, Beyler NK, Welk GJ, Carriquiry AL, Fuller WA, King BM. Modeling errors in physical activity recall data. J Phys Act Health. 2012;9:S56-S67. doi:10.1123/jpah.9.s1.s56

34. Carlson SA, Fulton JE, Schoenborn CA, Loustalot F. Trend and prevalence estimates based on the 2008 physical activity guidelines for Americans. Am J Prev Med. 2010;39(4):305-313. PubMed ID: 20837280 doi:10.1016/j.amepre.2010.06.006

35. Du Y, Liu B, Sun Y, Snetselaar LG, Wallace RB, Bao W. Trends in adherence to the physical activity guidelines for Americans for aerobic activity and time spent on sedentary behavior among US adults, 2007 to 2016. JAMA Netw Open. 2019;2(7):e197597. PubMed ID: 31348504 doi:10.1001/jamanetworkopen.2019. 7597

36. Reifschneider MJ, Hamrick KS, Lacey JN. Exercise, eating patterns, and obesity: evidence from the ATUS and its eating \& health module. Soc Indic Res. 2011;101(2):215-219. doi:10.1007/s11205010-9655-y

37. Saint-Maurice PF, Troiano RP, Bassett DR, et al. Association of daily step count and stepping intensity with mortality among US adults. JAMA. 2020;323(12):1151-1160. PubMed ID: 32207799 doi:10. 1001/jama.2020.1382

38. Tudor-Locke C, Johnson WD, Katzmarzyk PT. Frequently reported activities by intensity for US adults: the American time use survey. Am J Prev Med. 2010;39(4):e13-e20. PubMed ID: 20837277 doi:10. 1016/j.amepre.2010.05.017

39. Ham SA, Kruger J, Tudor-Locke C. Participation by US adults in sports, exercise, and recreational physical activities. J Phys Act Health. 2009;6(1):6-14. PubMed ID: 19211953 doi:10.1123/jpah.6.1.6

40. Harms T, Gershuny J, Doherty A, Thomas E, Milton K, Foster C. A validation study of the Eurostat harmonised European time use study (HETUS) diary using wearable technology. BMC Public Health. 2019;19:455. doi:10.1186/s12889-019-6761-x 
41. Kozey Keadle S, Lyden K, Hickey A, et al. Validation of a previous day recall for measuring the location and purpose of active and sedentary behaviors compared to direct observation. Int J Behav Nutr Phys Act. 2014;11:12. PubMed ID: 24490619 doi:10.1186/1479-5868-11-12

42. Community Preventive Services Task Force. Physical Activity. 2018. https://www.thecommunityguide.org/topic/physical-activity. Accessed February 5, 2019.

43. Matthews CE, Keadle SK, Sampson J, et al. Validation of a previousday recall measure of active and sedentary behaviors. Med Sci Sports Exerc. 2013;45(8):1629-1638. PubMed ID: 23863547 doi:10.1249/ MSS.0b013e3182897690

44. Adams SA, Matthews CE, Ebbeling CB, et al. The effect of social desirability and social approval on self-reports of physical activity.
Am J Epidemiol. 2005;161(4):389-398. PubMed ID: 15692083 doi:10.1093/aje/kwi054

45. Matthews CE, Freedson PS, Stanek EJ, et al. Seasonal variation of household, occupational, and leisure-time physical activity: longitudinal analyses from the seasonal variation of blood cholesterol study. Am J Epidemiol. 2001;153(2):172-183. doi:10.1093/aje/ 153.2.172

46. Arnardottir NY, Oskarsdottir ND, Brychta RJ, et al. Comparison of summer and winter objectively measured physical activity and sedentary behavior in older adults: age, gene/environment susceptibility reykjavik study. Int J Environ Res Public Health. 2017;14(10):1268. doi:10.3390/ijerph14101268 